\title{
Motion of Level Sets by Mean Curvature III
}

\author{
By L.C. Evans and J. Spruck
}

\begin{abstract}
We continue our investigation [6,7] (see also [4], etc.) of the generalized motion of sets via mean curvature by the level set method. We study more carefully the fine properties of the mean curvature PDE, to obtain Hausdorff measure estimates of level sets and smoothness whenever the level sets are graphs.
\end{abstract}

\section{Introduction}

This paper continues our investigation $[6,7]$ of a generalized motion by mean curvature of compact sets in $\mathbb{R}^{n}$.

Since a classical such motion starting with a smooth hypersurface $\Gamma_{0}$ will in general develop singularities after a finite time, the primary goal of our previous paper [6] was to define and construct a reasonable generalized evolution $\left\{\Gamma_{t}\right\}_{t>0}$, which exists for all time and agrees with the classical flow so long as the latter exists. Following Osher and Sethian $[15,16]$, we accomplish this as follows: First, given $\Gamma_{0}$ as above we select a smooth function $g: \mathbb{R}^{n} \rightarrow \mathbb{R}$, with $\Gamma_{0}=\left\{x \in \mathbb{R}^{n} \mid g(x)=0\right\}$. We next construct the (appropriately defined) weak solution $u: \mathbb{R}^{n} \times[0, \infty) \rightarrow \mathbb{R}$ of the nonlinear PDE

$$
\left\{\begin{array}{l}
u_{t}=\left(\delta_{i j}-\frac{u_{x_{i}} u_{x_{j}}}{|D u|^{2}}\right) u_{x_{i} x_{j}} \text { in } \mathbb{R}^{n} \times(0, \infty) \\
u=g \text { on } \mathbb{R}^{n} \times\{t=0\} .
\end{array}\right.
$$

Math Subject Classification 53A10, 53A99, 35K55.

Key Words and Phrases Evolution by mean curvature, Hausdorff measure, weak solutions of nonlinear PDE.

L. C. E. was supported in part by NSF Grant DMS-86-01532. J. S. was supported in part by NSF Grant DMS-8802858 and DOE Grant DE-FG02-86ER25015. 
As noted in [6], this equation asserts that each level set of $u$ evolves by mean curvature motion, at least in regions where $u$ is smooth and $D u \neq 0$. Consequently, it seems reasonable to define $\Gamma_{t} \equiv\left\{x \in \mathbb{R}^{n} \mid u(x, t)=0\right\}$ for each time $t \geq 0$. The collection of sets $\left\{\Gamma_{t}\right\}_{t \geq 0}$ comprises our generalized evolution by mean curvature. (Chen, Giga, and Goto [4] simultaneously and independently developed a similar approach applied to more general geometric motions.)

The generalized evolution $\left\{\Gamma_{t}\right\}_{t \geq 0}$ in hand, the primary task is then to study the geometric properties of the flow $\Gamma_{0} \mapsto \Gamma_{t}(t \geq 0)$. This undertaking is particularly pressing in view of the important and much earlier work of Brakke [2], who also utilized varifold methods from geometric measure theory to construct generalized mean curvature motions. Unfortunately, Brakke's evolutions do not in general agree with that described above: see [6, Sec. 8] for a preliminary discussion of this point.

Our purpose in this paper is to effect at least a partial reconciliation between the viewpoints of $[6,4]$ and $[2]$ by applying geometric measure theory techniques to analyze the structure of our generalized evolution $\left\{\Gamma_{t}\right\}_{t \geq 0}$.

This study we carry out in several steps. In Section 2 we first make some simple calculations that allow us to invoke standard GMT assertions to establish that for a.e. $\gamma \in \mathbb{R}$ the level sets $\Gamma_{t}^{\gamma} \equiv\left\{x \in \mathbb{R}^{n} \mid u(x, t)=\gamma\right\}$ are countably $H^{n-1}$-rectifiable for a.e. $t>0$. Here $H^{n-1}$ denotes $(n-1)$-dimensional Hausdorff measure in $\mathbb{R}^{n}$. This general assertion is not very enlightening as we are interested only in the particular level set $\Gamma_{t}=\Gamma_{t}^{0}$ corresponding to $\gamma=0$. We require more refined tools.

Consequently, in Section 3 we establish for our generalized motion an analog of Brakke's important "clearing out" lemma [2, Sec. 6.3]. This asserts that if at some time $t_{0}$ the $(n-1)$ dimensional Hausdorff measure of $\Gamma_{t_{0}}$ within a ball is sufficiently small, then $\Gamma_{t}$ does not intersect a smaller concentric ball for certain later times $t>t_{0}$. Our proof utilizes approximations by smooth motions via mean curvature in one more dimension (cf. [6, Sec. 4.1]).

An immediate consequence in Section 4 is an estimate of the extinction time of $\left\{\Gamma_{t}\right\}_{t>0}$ in terms of $H^{n-1}\left(\Gamma_{0}\right)$. A further and somewhat more subtle application asserts that $H^{n-1}\left(\Gamma_{t}^{*}\right)<\infty$ for each time $t>0$, provided $\Gamma_{0}$ is compact, $H^{n-1}$-rectifiable, with $H^{n-1}\left(\Gamma_{0}\right)<\infty$. Here $\Gamma_{t}^{*}=\partial \Gamma_{t}$, the topological boundary of $\Gamma_{t}$.

Finally, in Section 5 we demonstrate that if some part of our generalized evolution can be written as a graph, then that portion is in fact a smooth hypersurface moving by mean curvature. This is a first regularity assertion for our generalized flow. As a corollary of this result, we show (Theorem 5.5) that an initial surface consisting of the boundary of an arbitrary convex set becomes smooth and convex. We hope in future work to show that in general $\left\{\Gamma_{t}^{*}\right\}_{t \geq 0}$ is smooth, except for a "small" singular set.

We thank the referee for a careful reading of this paper, and particularly for suggesting a simplification of the proof of Lemma 2.3. Also, we have recently seen a new paper of Ecker and Huisken that provides local gradient bounds for graphs moving via mean curvature. This work is strongly related to the estimates we obtain in Section 5 . 


\section{Hausdorff measure of a.e. level set}

Choose $g: \mathbb{R}^{n} \rightarrow \mathbb{R}$ to be smooth, bounded, and constant outside some ball. As in [6, Sec. 4.1] we introduce for each $\epsilon>0$ the approximating PDE

$$
\left\{\begin{array}{l}
u_{t}^{\epsilon}=\left(\delta_{i j}-\frac{u_{x_{i}}^{\epsilon} u_{x_{j}}^{\epsilon}}{\left|D u^{\epsilon}\right|^{2}+\epsilon^{2}}\right) u_{x_{i} x_{j}}^{\epsilon} \text { in } \mathbb{R}^{n} \times[0, \infty) \\
u^{\epsilon}=g \text { on } \mathbb{R}^{n} \times\{t=0\} .
\end{array}\right.
$$

This quasilinear parabolic initial value problem has a unique, bounded smooth solution $u^{\epsilon}$. According to [6, Sec. 4.2], we additionally have the bounds

$$
\sup _{0<\epsilon<1}\left\|u^{\epsilon}, D u^{\epsilon}, u_{t}^{\epsilon}\right\|_{L^{\infty}\left(R^{n} \times(0, \infty)\right)}<\infty
$$

Furthermore, as $\epsilon \rightarrow 0$,

$$
\begin{cases}u^{\epsilon} \rightarrow u & \text { locally uniformly on }\left(\mathbb{R}^{n} \times[0, \infty)\right. \\ D u^{\epsilon} \stackrel{*}{\rightarrow} D u, u_{t}^{\epsilon} \stackrel{*}{\rightarrow} u_{t} & \text { weakly-* in } L^{\infty}\left(\mathbb{R}^{n} \times[0, \infty)\right)\end{cases}
$$

$u$ denoting the unique weak solution of the mean curvature evolution PDE

$$
\left\{\begin{array}{l}
u_{t}=\left(\delta_{i j}-\frac{u_{x_{i}} u_{x_{j}}}{|D u|^{2}}\right) u_{x_{i} x_{j}} \quad \text { in } \mathbb{R}^{n} \times[0, \infty) \\
u=g \text { on } \mathbb{R}^{n} \times\{t=0\}
\end{array}\right.
$$

See $\left[6\right.$, Sec. 2] for the definition of a weak solution. Note that $u: \mathbb{R}^{n} \times[0, \infty) \rightarrow \mathbb{R}$ is bounded, Lipschitz, and constant outside some compact set.

Lemma 2.1. We have the estimate

$$
\sup _{t \geq 0} \int_{R^{n}}|D u| d x \leq \int_{R^{n}}|D g| d x
$$

Proof. Fix $\delta>0$ and set

$$
\begin{aligned}
\phi(x) & =\phi_{\delta}(x) \equiv e^{-\delta\left(1+|x|^{2}\right)^{1 / 2}} \quad\left(x \in \mathbb{R}^{n}\right), \\
\Phi^{\epsilon, \delta}(t) & \equiv \int_{R^{n}} \phi^{2}\left(\left|D u^{\epsilon}\right|^{2}+\epsilon^{2}\right)^{1 / 2} d x .
\end{aligned}
$$


We then compute

$$
\begin{aligned}
\left(\Phi^{\epsilon, \delta}\right)^{\prime}(t) & =\int_{R^{n}} \phi^{2} \frac{D u^{\epsilon} \cdot D u_{t}^{\epsilon}}{\left(\left|D u^{\epsilon}\right|^{2}+\epsilon^{2}\right)^{1 / 2}} d x \\
& =-\int_{R^{n}} \phi^{2} \operatorname{div}\left(\frac{D u^{\epsilon}}{\left(\left|D u^{\epsilon}\right|^{2}+\epsilon^{2}\right)^{1 / 2}}\right) u_{t}^{\epsilon}+2 \phi \frac{D \phi \cdot D u^{\epsilon}}{\left(\left|D u^{\epsilon}\right|^{2}+\epsilon^{2}\right)^{1 / 2}} u_{t}^{\epsilon} d x \\
& =-\int_{R^{n}} \phi^{2}\left(H^{\epsilon}\right)^{2}\left(\left|D u^{\epsilon}\right|^{2}+\epsilon^{2}\right)^{1 / 2}+2 \phi D \phi \cdot D u^{\epsilon} H^{\epsilon} d x \quad \text { by (2.1), }
\end{aligned}
$$

where

$$
H^{\epsilon} \equiv \operatorname{div}\left(\frac{D u^{\epsilon}}{\left(\left|D u^{\epsilon}\right|^{2}+\epsilon^{2}\right)^{1 / 2}}\right)
$$

Thus

$$
\begin{aligned}
\left(\Phi^{\epsilon, \delta}\right)^{\prime}(t) & \leq \int_{R^{n}}|D \phi|^{2}\left(\left|D u^{\epsilon}\right|^{2}+\epsilon^{2}\right)^{1 / 2} d x \\
& \leq \delta^{2} \Phi^{\epsilon, \delta}(t) \quad(t \geq 0)
\end{aligned}
$$

since (2.5) implies $|D \phi|^{2} \leq \delta^{2} \phi^{2}$. Applying Gronwall's inequality we obtain

$$
\begin{aligned}
\left.\int_{R^{n}} \phi^{2}\left|D u^{\epsilon}\right| d x\right|_{t=T} & \leq\left.\int_{R^{n}} \phi^{2}\left(\left|D u^{\epsilon}\right|^{2}+\epsilon^{2}\right)^{1 / 2} d x\right|_{t=T} \\
& \leq e^{\delta^{2} T} \int_{R^{n}} \phi^{2}\left(|D g|^{2}+\epsilon^{2}\right)^{1 / 2} d x
\end{aligned}
$$

We recall (2.2) and then send $\epsilon, \delta \rightarrow 0$ to deduce

$$
\left.\int_{R^{n}}|D u| d x\right|_{t=T} \leq \int_{R^{n}}|D g| d x
$$

for each time $T>0$.

For each real number $\gamma \in \mathbb{R}$ and time $t \geq 0$, let us define

$$
\Gamma_{t}^{\gamma} \equiv\left\{x \in \mathbb{R}^{n} \mid u(x, t)=\gamma\right\}
$$

We now utilize estimate (2.4) to study the measure theoretic structure of the level sets $\Gamma_{t}^{\gamma}$ for almost every $(\gamma, t)$. We utilize the terminology of Federer [8, Sec. 3.2.14].

Lemma 2.2. (i) For a.e. $(\gamma, t) \in \mathbb{R}^{n} \times[0, \infty)$, $\Gamma_{t}^{\gamma}$ is $\left(H^{n-1}, n-1\right)$-rectifiable. In particular, for a.e. $\gamma \in \mathbb{R}, \Gamma_{t}^{\gamma}$ is $\left(H^{n-1}, n-1\right)$-rectifiable for a.e. $t \geq 0$. 
(ii) For a.e. $\gamma \in \mathbb{R}$, we have the estimate

$$
\sup _{0 \leq t<\infty} H^{n-1}\left(\Gamma_{t}^{\gamma}\right) \leq H^{n-1}\left(\Gamma_{0}^{\gamma}\right)<\infty
$$

Proof. (1) Define the function $U: \mathbb{R}^{n} \times[0, \infty) \rightarrow \mathbb{R}^{2}$ by

$$
U(x, t)=(u(x, t), t) \quad\left(x \in \mathbb{R}^{n}, t \geq 0\right) .
$$

Then $U$ is Lipschitz, whence Federer [8, Sec. 3.2.15] implies $\Gamma_{t}^{\gamma}$ is countably $\left(H^{n-1}, n-1\right)$ rectifiable for a.e. $(\gamma, t)$.

In addition, the mapping $(\gamma, t) \mapsto H^{n-1}\left(\Gamma_{t}^{\gamma}\right)$ is measurable; see Federer [8, Sec. 2.10.26].

(2) Assume $\Psi: \mathbb{R} \rightarrow \mathbb{R}$ is smooth and nondecreasing. According to [6, Sec. 2.4], $\tilde{u} \equiv \Psi(u)$ is the unique weak solution of the mean curvature evolution PDE (2.3) corresponding to the initial data $\tilde{g} \equiv \Psi(g)$. Thus Lemma 2.1 (with $\tilde{u}, \tilde{g}$ replacing $u, g$ ) provides the bound

$$
\int_{R^{n}} \Psi^{\prime}(u)|D u| d x \leq \int_{R^{n}} \Psi^{\prime}(g)|D g| d x
$$

for each $t>0$. Approximating, we deduce

$$
\frac{1}{h} \int_{\{\gamma<u<\gamma+h\}}|D u| d x \leq \frac{1}{h} \int_{\{\gamma<g<\gamma+h\}}|D g| d x
$$

for each $\gamma \in \mathbb{R}, h>0$, and $t>0$. Using the Coarea Formula, we rewrite this inequality to read

$$
\frac{1}{h} \int_{\gamma}^{\gamma+h} H^{n-1}\left(\Gamma_{t}^{\gamma}\right) d \gamma \leq \frac{1}{h} \int_{\gamma}^{\gamma+h} H^{n-1}\left(\Gamma_{0}^{\gamma}\right) d \gamma
$$

Integrating in $t$ yields

$$
\frac{1}{h^{2}} \int_{t}^{t+h} \int_{\gamma}^{\gamma+h} H^{n-1}\left(\Gamma_{s}^{\gamma}\right) d \gamma d s \leq \frac{1}{h} \int_{\gamma}^{\gamma+h} H^{n-1}\left(\Gamma_{0}^{\gamma}\right) d \gamma .
$$

As the integrand on the left-hand side is summable, we deduce upon sending $h \rightarrow 0^{+}$that

$$
H^{n-1}\left(\Gamma_{t}^{\gamma}\right) \leq H^{n-1}\left(\Gamma_{0}^{\gamma}\right)
$$

for a.e. $(\gamma, t)$.

For later use, we record the following lemma. 
Lemma 2.3. Let $K \subset \mathbb{R}^{n}$ be compact, $0<H^{n-1}(K)<\infty$. Then there exists a smooth, open, bounded set $V \supset K$ such that

$$
H^{n-1}(\partial V) \leq C H^{n-1}(K)
$$

the constant $C$ depending only on $n$.

Proof. (1) By compactness and the definition of $H^{n-1}$ there are finitely many open balls $\left\{U\left(x_{i}, r_{i}\right)\right\}_{i=1}^{N}$ so that

$$
K \subset \bigcup_{i=1}^{N} U\left(x_{i}, r_{i}\right) \equiv U
$$

and

$$
\sum_{i=1}^{N} r_{i}^{n-1} \leq C H^{n-1}(K)
$$

Then

$$
\partial U \subseteq \bigcup_{i=1}^{N} \partial U\left(x_{i}, r_{i}\right)
$$

whence

$$
H^{n-1}(\partial U) \leq C \sum_{i=1}^{N} r_{i}^{n-1} \leq C H^{n-1}(K)
$$

(2) Denote by $\Delta$ the regularized distance function to $U$ constructed in Stein [18, Sec. 1.2.1]. Then

$$
\begin{aligned}
\left|\left\{x \in \mathbb{R}^{n} \mid \Delta(x)<r\right\}\right| & \leq\left|\left\{x \in \mathbb{R}^{n} \mid \operatorname{dist}(x, U) \leq \mu r\right\}\right| \\
& \leq \sum_{i=1}^{N}\left|U\left(x_{i}, r_{i}+\mu r\right)-U\left(x_{i}, r_{i}\right)\right| \leq C r \sum_{i=1}^{N} r_{i}^{n-1}
\end{aligned}
$$

for some constant $\mu>0$ and all $r$ small enough. The Coarea Formula implies

$$
\begin{aligned}
\int_{0}^{r} H^{n-1}(\{\Delta=s\}) d s & =\int_{\{0<\Delta<r\}}|D \Delta| d x \leq C\left|\left\{x \in \mathbb{R}^{n} \mid \Delta(x)<r\right\}\right| \\
& \leq C r \sum_{i=1}^{N} r_{i}^{n-1} \leq C r H^{n-1}(K), \text { by (2.10). }
\end{aligned}
$$

Since $\Delta$ is $C^{\infty},(2.11)$ and Sard's Theorem imply the existence of $0<s<r$ such that $V \equiv\left\{x \in \mathbb{R}^{n} \mid \Delta(x)<s\right\}$ is smooth and $H^{n-1}(\partial V) \leq C H^{n-1}(K)$. 


\section{Clearing out}

In this section we prove an analog for our generalized mean curvature flow of Brakke's fundamental "clearing out" lemma [2, Sec. 6.3]. This asserts that if at time $t_{0} \geq 0$ there is only a "small amount" of $\Gamma_{t_{0}}$ within some ball, then for certain later times $t>t_{0}, \Gamma_{t}$ does not intersect a smaller concentric ball at all.

Theorem 3.1. There exist constants $\alpha, \beta, \eta>0$ such that if

$$
H^{n-1}\left(\Gamma_{t_{0}} \cap B\left(x_{0}, r\right)\right) \leq \eta r^{n-1}
$$

for some time $t_{0} \geq 0$ and some ball $B\left(x_{0}, r\right) \subset \mathbb{R}^{n}$, then

$$
\Gamma_{t} \cap B\left(x_{0}, \frac{r}{2}\right)=\emptyset \text { for } \alpha r^{2} \leq t-t_{0} \leq \beta r^{2}
$$

Remark. We will see in the course of the proof (cf. 3.12) that we can take $\alpha>0$ as small as we wish, provided we adjust $\eta>0$ to be sufficiently small. Hence we may additionally assume

$$
0<\alpha<\frac{\beta}{4}
$$

This inequality will be useful in a forthcoming paper.

Proof. Our proof utilizes several key ideas of Brakke [2].

(1) We may, upon translating and rescaling, suppose $t_{0}=0, x_{0}=0, r=1$. Thus we are assuming

$$
H^{n-1}\left(\Gamma_{0} \cap B(0,1)\right) \leq \eta
$$

$\eta>0$ to be selected.

(2) Step 1. Classical mean curvature motion. For the moment, let us also suppose $\left\{\Gamma_{t}\right\}_{t \geq 0}$ is a classical flow by mean curvature and $n \geq 3$. Given a $C^{2}$ function $f: \mathbb{R}^{n} \times[0, \infty) \rightarrow[0,1]$ with compact support, set

$$
\Phi(t) \equiv \int_{\Gamma_{t}} f(x, t) d H^{n-1}(x) \quad(t \geq 0)
$$

A classic calculation (cf. Huisken [10]) shows

$$
\Phi^{\prime}(t)=\int_{\Gamma_{t}}\left(f_{t}-\left(\delta_{i j}-\nu_{i} \nu_{j}\right) f_{x_{i} x_{j}}-f H^{2}\right) d H^{n-1}
$$


$\nu=\left(\nu_{1}, \ldots, \nu_{n}\right)$ denoting a unit normal vector field to $\Gamma_{t}$ and $H$ is $(n-1)$ times the mean curvature, computed with respect to $\nu$. Write

$$
f(x, t) \equiv h\left(1-|x|^{2}-\mu t\right) \quad\left(x \in \mathbb{R}^{n}, t \geq 0\right),
$$

the function $h$ and the constant $\mu>0$ to be selected below. Then plugging in above we discover

$$
\begin{aligned}
\Phi^{\prime}(t) & =\int_{\Gamma_{i}}\left\{-\mu h^{\prime}-\left(\delta_{i j}-\nu_{i} \nu_{j}\right)\left(-2 \delta_{i j} h^{\prime}+4 x_{i} x_{j} h^{\prime \prime}\right)-h H^{2}\right\} d H^{n-1} \\
& =\int_{\Gamma_{i}}\left\{h\left(-H^{2}\right)+h^{\prime}(-\mu+2(n-1))+h^{\prime \prime} 4\left((x \cdot \nu)^{2}-|x|^{2}\right)\right\} d H^{n-1} .
\end{aligned}
$$

Taking $h$ to be nonnegative, convex, and nondecreasing, and setting $\mu \equiv 2 n-1$, we obtain

$$
\Phi^{\prime}(t) \leq-\int_{\Gamma_{t}}\left(H^{2} h+h^{\prime}\right) d H^{n-1} \leq 0
$$

(3) Since $f$ has compact support, we may invoke the Sobolev-type inequality

$$
\left(\int_{\Gamma_{t}} f^{n-1 / n-2} d H^{n-1}\right)^{\frac{n-2}{n-1}} \leq C \int_{\Gamma_{t}}(|D f|+|f H|) d H^{n-1}
$$

(See, for instance, Michael and Simon [14] or Burago and Zalgaller [3, Sec. 28.5.2].)

Now set

$$
h(z) \equiv \begin{cases}z^{3} & z \geq 0 \\ 0 & z \leq 0\end{cases}
$$

Then $h$ is $C^{2}$, convex, and

$$
h^{\prime}=3 h^{2 / 3} \geq 0
$$

We compute

$$
\begin{aligned}
\Phi(t) & =\int_{\Gamma_{t}} f d H^{n-1} \\
& \leq\left(\int_{\Gamma_{t}} f^{\theta r} d H^{n-1}\right)^{1 / r}\left(\int_{\Gamma_{t}} f^{(1-\theta) s} d H^{n-1}\right)^{1 / s}
\end{aligned}
$$


where $0<\theta<1,1<r, s<\infty, 1 / r+1 / s=1$. Setting

$$
\theta=\frac{n-1}{n+1}, \quad r=\frac{n+1}{n-2}, \quad s=\frac{n+1}{3}
$$

yields

$$
\begin{aligned}
\Phi(t) & \leq\left(\int_{\Gamma_{t}} f^{\frac{n-1}{n-2}} d H^{n-1}\right)^{\frac{n-2}{n+1}}\left(\int_{\Gamma_{t}} f^{2 / 3} d H^{n-1}\right)^{3 / n+1} \\
& \leq C\left(\int_{\Gamma_{t}}|D f|+|f H| d H^{n-1}\right)^{\frac{n-1}{n+1}}\left(\int_{\Gamma_{t}} f^{2 / 3} d H^{n-1}\right)^{3 / n+1} \text { by (3.8) } \\
& \leq C\left(\int_{\Gamma_{t}} h^{\prime}+H^{2} h d H^{n-1}\right)^{\frac{n-1}{n+1}}\left(\int_{\Gamma_{t}} h^{\prime} d H^{n-1}\right)^{3 / n+1} \text { by (3.6), (3.9) } \\
& \leq C\left(-\Phi^{\prime}(t)\right)^{\frac{n+2}{n+1}} \text { by (3.7). }
\end{aligned}
$$

Here we have used (3.9) to estimate

$$
|f H| \leq h H \leq h+h H^{2} \leq C\left(h^{\prime}+h H^{2}\right) .
$$

Rewriting, we deduce

$$
\Phi^{\prime}(t) \leq-C \Phi(t)^{\frac{n+1}{n+2}} \quad(t \geq 0)
$$

whence

$$
\Phi(t)=0 \quad \text { for } \quad t>C \Phi(0)^{\frac{1}{n+2}} .
$$

Now

$$
f \geq h\left(1-(7 / 8)^{2}-\mu t\right)>0 \quad \text { on } \quad B(0,7 / 8)
$$

for $0 \leq t \leq \beta, \beta$ small enough. In addition,

$$
\Phi(0) \leq H^{n-1}\left(\Gamma_{0} \cap B(0,1)\right) \leq \eta
$$

owing to (3.3). Choose $\eta>0$ so small that

$$
\alpha \equiv C \eta^{\frac{1}{n+2}}<\beta
$$

the constant $C$ taken from (3.10). Then (3.10), (3.11) imply

$$
H^{n-1}\left(\Gamma_{t} \cap B(0,7 / 8)\right)=0 \quad(\alpha \leq t \leq \beta) .
$$


Since $\Gamma_{t}$ is a smooth hypersurface, this forces

$$
\Gamma_{t} \cap B(0,3 / 4)=\emptyset \quad(\alpha \leq t \leq \beta)
$$

(4) Step 2: $\Gamma_{0}$ smooth. Next assume $\Gamma_{0}$ is the boundary of a smooth, bounded open set $U \subset \mathbb{R}^{n}, n \geq 2$. Choose a smooth function $g: \mathbb{R}^{n} \rightarrow \mathbb{R}$ with $\Gamma_{0}=\{g=0\}, D g \neq 0$ on $\Gamma_{0}$.

Fix $\epsilon>0$ and consider the smooth solution $u^{\epsilon}$ of the approximating PDE (2.1). As in [6, Sec. 4.1], define

$$
\left\{\begin{array}{l}
v^{\epsilon}(y, t) \equiv u^{\epsilon}(x, t)-\epsilon x_{n+1} \\
g^{\epsilon}(y) \equiv g(x)-\epsilon x_{n+1}
\end{array}\right.
$$

for $t \geq 0, y=\left(x, x_{n+1}\right) \in \mathbb{R}^{n+1}$. Then $v^{\epsilon}$ satisfies the mean curvature PDE

$$
\begin{cases}v_{t}^{\epsilon}=\left(\delta_{i j}-\frac{v_{y_{i}}^{\epsilon} v_{y_{j}}^{\epsilon}}{\left|D v^{\epsilon}\right|^{2}}\right) v_{y_{i} y_{j}}^{\epsilon} & \text { in } \mathbb{R}^{n+1} \times[0, \infty) \\ v^{\epsilon}=g^{\epsilon} & \text { on } \mathbb{R}^{n+1} \times\{t=0\}\end{cases}
$$

(The implicit summation is from 1 to $n+1$ here.)

In particular, the level sets

$$
\begin{aligned}
\Gamma_{t}^{\epsilon} & =\left\{y \in \mathbb{R}^{n+1} \mid v^{\epsilon}(y, t)=0\right\} \\
& =\left\{y=\left(x, x_{n+1}\right) \in \mathbb{R}^{n+1} \mid x_{n+1}=\frac{1}{\epsilon} u^{\epsilon}(x, t)\right\}
\end{aligned}
$$

are smooth entire graphs evolving via mean curvature motion in $\mathbb{R}^{n+1}$, starting from the initial surface

$$
\Gamma_{0}^{\epsilon} \equiv\left\{y=\left(x, x_{n+1}\right) \in \mathbb{R}^{n+1} \mid x_{n+1}=\frac{1}{\epsilon} g(x)\right\}
$$

(cf. Ecker and Huisken [5]). Consequently, the argument in step 1 above (with $n+1$ replacing $n$ ) allows us to conclude

$$
\Gamma_{t}^{\epsilon} \cap B^{n+1}(0,3 / 4)=\emptyset \quad(\alpha \leq t \leq \beta)
$$


for appropriate constants $0<\alpha<\beta$, provided

$$
\Phi^{\epsilon}(0)=\int_{\Gamma_{0}^{\epsilon}} f d H^{n}
$$

is small enough. Here

$$
f(y, t)=h\left(1-|y|^{2}-\mu t\right), y=\left(x, x_{n+1}\right),
$$

and $B^{n+1}(0, r)$ denotes the closed ball in $\mathbb{R}^{n+1}$ with center 0 , radius $r$. Now

$$
\begin{aligned}
\Phi^{\epsilon}(0) & \leq H^{n}\left(\Gamma_{0}^{\epsilon} \cap B^{n+1}(0,1)\right) \\
& \leq H^{n}\left(\Gamma_{0}^{\epsilon} \cap(B(0,1) \times[-1,1])\right) \\
& =\int_{B(0,1) \cap\{(1 / \epsilon)|g| \leq 1\}}\left(1+\frac{1}{\epsilon^{2}}|D g|^{2}\right)^{1 / 2} d x \text { by (3.16) } \\
& =\frac{1}{\epsilon} \int_{B(0,1) \cap\{|g| \leq \epsilon\}}\left(\epsilon^{2}+|D g|^{2}\right)^{1 / 2} d x
\end{aligned}
$$

Since $\Gamma_{0}$ is smooth and $D g \neq 0$ on $\Gamma_{0}$, we see

$$
\begin{aligned}
\Phi^{\epsilon}(0) & \leq 2 H^{n-1}\left(\Gamma_{0} \cap B(0,1)\right)+o(1) \quad \text { as } \epsilon \rightarrow 0 \\
& \leq 2 \eta+o(1) \\
& \leq 3 \eta
\end{aligned}
$$

for $0<\epsilon<\epsilon_{0}, \epsilon_{0}$ small enough. Consequently, (3.17) obtains if $\eta$ is small enough and $0<\epsilon<$ $\epsilon_{0}$.

(5) More generally now, if $\gamma \in \mathbb{R}$, let us write

$$
\begin{aligned}
\Gamma_{t}^{\epsilon, \gamma} & =\left\{y \in \mathbb{R}^{n+1} \mid v^{\epsilon}(y, t)=\gamma\right\} \\
& =\left\{y=\left(x, x_{n+1}\right) \in \mathbb{R}^{n+1} \mid x_{n+1}=\frac{1}{\epsilon}\left(u^{\epsilon}(x, t)-\gamma\right)\right\}
\end{aligned}
$$

and

$$
\Gamma_{0}^{\gamma}=\left\{x \in \mathbb{R}^{n} \mid g(x)=\gamma\right\}
$$

As $\Gamma_{0}$ and $g$ are smooth, we may assume

$$
H^{n-1}\left(\Gamma_{0}^{\gamma}\right) \leq C H^{n-1}\left(\Gamma_{0}\right)
$$

provided $|\gamma|$ is small enough. 
Applying the argument above with the evolution $\left\{\Gamma_{t}^{\epsilon, \gamma}\right\}_{t \geq 0}$ replacing $\left\{\Gamma_{t}^{\epsilon}\right\}_{t \geq 0}$, we deduce

$$
\Gamma_{t}^{\epsilon, \gamma} \cap B^{n+1}(0,3 / 4)=\emptyset \quad(\alpha \leq t \leq \beta)
$$

for appropriate $0<\alpha<\beta$, provided $0 \leq|\gamma| \leq \gamma_{0}, 0<\epsilon \leq \epsilon_{0}$, and (3.4) holds for sufficiently small $\eta$. Thus

$$
\left|u^{\epsilon}\right| \geq \gamma_{0} \text { on } B(0,3 / 4) \quad(\alpha \leq t \leq \beta)
$$

if $0<\epsilon<\epsilon_{0}$. Letting $\epsilon \rightarrow 0$, we deduce

$$
|u| \geq \gamma_{0} \text { on } B(0,3 / 4) \quad(\alpha \leq t \leq \beta)
$$

Consequently,

$$
\Gamma_{t} \cap B(0,3 / 4)=\emptyset \quad(\alpha \leq t \leq \beta)
$$

(6) Step 3: Arbitrary compact $\Gamma_{0}$. Finally, suppose only that $\Gamma_{0} \subset \mathbb{R}^{n}$ is compact, with (3.4) holding.

We apply Lemma 2.3 to the compact set $K \equiv \Gamma_{0} \cap B(0,7 / 8)$ to obtain a smooth open set $V \supset K$ satisfying

$$
H^{n-1}(\partial V) \leq C H^{n-1}(K) \leq C \eta
$$

Set

$$
\Delta_{0} \equiv \overline{V \bigcup\left(\mathbb{R}^{n}-B(0,7 / 8)\right)}
$$

Then

$$
\Delta_{0} \supseteq \Gamma_{0}
$$

and so [6, Theorem 7.2] implies

$$
\Delta_{t} \supseteq \Gamma_{t}
$$

$\left\{\Delta_{t}\right\}_{t \geq 0}$ denoting the generalized mean curvature motion starting with $\Delta_{0}$. We may as well also suppose

$$
\hat{\Gamma}_{0} \equiv \partial \Delta_{0} \text { is smooth; }
$$

for if not we can enlarge $V$ within the annulus $B(0,7 / 8)-B(0,3 / 4)$ to achieve (3.23). 
We turn our attention to the ball $B(0,3 / 4)$ and observe from $(3.20),(3.21)$ that

$$
H^{n-1}\left(\hat{\Gamma}_{0} \cap B(0,3 / 4)\right) \leq H^{n-1}(\partial V) \leq C \eta
$$

Let $\left\{\Gamma_{t}\right\}_{t \geq 0}$ denote the mean curvature flow starting with $\Gamma_{0}$. Then if $\eta$ is small enough, step 2 implies

$$
\hat{\Gamma}_{t} \cap B(0,1 / 2)=\emptyset \quad(\alpha \leq t \leq \beta)
$$

for appropriate constants $0<\alpha<\beta$.

(7) Choose a smooth function $g$ satisfying

$$
\left\{\begin{array}{l}
g=0 \text { on } \hat{\Gamma}_{0} \\
g<0 \text { in the interior of } \Delta_{0} \\
g>0 \text { in } \mathbb{R}^{n}-\Delta_{0}
\end{array}\right.
$$

Then

$$
\left\{\begin{array}{l}
g^{+}=0 \text { in } \Delta_{0} \\
g^{+}>0 \text { in } \mathbb{R}^{n}-\Delta_{0}
\end{array}\right.
$$

Let $u$ denote the weak solution of the mean curvature PDE (2.1) corresponding to the initial function $g$. From [6, Theorem 2.8] we recall $u^{+}$is the (unique) weak solution of (2.1) corresponding to the initial function $g^{+}$. Now

$$
\hat{\Gamma}_{t}=\left\{x \in \mathbb{R}^{n} \mid u(x, t)=0\right\}
$$

and

$$
\begin{aligned}
\Delta_{t} & =\left\{x \in \mathbb{R}^{n} \mid u^{+}(x, t)=0\right\} \\
& =\left\{x \in \mathbb{R}^{n} \mid u(x, t) \leq 0\right\} .
\end{aligned}
$$

In view of (3.24) we have $u \neq 0$ on $B(0,1 / 2) \times[\alpha, \beta]$; and so (3.26) allows us to infer either

$$
\Delta_{t} \cap B(0,1 / 2)=\emptyset \quad(\alpha \leq t \leq \beta)
$$

or

$$
\Delta_{t} \cap B(0,1 / 2)=B(0,1 / 2) \quad(\alpha \leq t \leq \beta) .
$$


Lemma 3.2 below excludes the latter possibility provided $\eta$ is small enough. Consequently, (3.27) must hold; whence (3.27) implies

$$
\Gamma_{t} \cap B(0,1 / 2)=\emptyset \quad(\alpha \leq t \leq \beta),
$$

as desired.

Lemma 3.2. Statement (3.28) is impossible if $\eta$ is sufficiently small.

Proof. Let $u^{\epsilon}$ be the smooth solution of the approximating PDE (2.1) corresponding to the initial function $g$ satisfying (3.25).

(1) Define $f(x, t) \equiv h\left(7 / 8-|x|^{2}-\mu t\right)$ (similarly to step 1 of the preceding proof) and

$$
\Phi_{1}^{\epsilon}(t) \equiv \int_{R^{n}} f\left(\left|D u^{\epsilon}\right|^{2}+\epsilon^{2}\right)^{1 / 2} d x
$$

A calculation shows

$$
\left(\Phi_{1}^{\epsilon}\right)^{\prime}(t) \leq 0 \quad(t \geq 0)
$$

(cf. inequality (3.7) above). Since

$$
f \geq \sigma>0 \quad \text { on } \quad B(0,2 / 3) \times[0, \beta]
$$

for some $\sigma$, if $\mu$ is large enough, and $\beta$ small enough, we deduce upon integrating that

$$
\sup _{0 \leq t \leq \beta} \int_{B(0,2 / 3)}\left(\left|D u^{\epsilon}\right|^{2}+\epsilon^{2}\right)^{1 / 2} d x \leq C \int_{B(0,3 / 4)}\left(|D g|^{2}+\epsilon^{2}\right)^{1 / 2} d x .
$$

(2) Choose now a smooth cutoff function $\zeta: \mathbb{R}^{n} \rightarrow \mathbb{R}$ satisfying

$$
\begin{cases}0 \leq \zeta \leq 1 & \\ \zeta \equiv 1 & \text { on } B(0,1 / 2) \\ \zeta \equiv 0 & \text { on } \mathbb{R}^{n}-B(0,2 / 3) .\end{cases}
$$

Then, setting

$$
\Phi_{2}^{\epsilon}(t) \equiv \int_{R^{n}} \zeta^{2}\left(\left|D u^{\epsilon}\right|^{2}+\epsilon^{2}\right)^{1 / 2} d x
$$

we compute similarly to the calculation in the proof of the Lemma 2.1 that

$$
\left(\Phi_{2}^{\epsilon}\right)^{\prime}(t)+\frac{1}{2} \int_{R^{n}} \zeta^{2}\left(H^{\epsilon}\right)^{2}\left(\left|D u^{\epsilon}\right|^{2}+\epsilon^{2}\right)^{1 / 2} d x \leq 4 \int_{R^{n}}|D \zeta|^{2}\left(\left|D u^{\epsilon}\right|^{2}+\epsilon^{2}\right)^{1 / 2} d x
$$


Integrating and recalling from (2.1) that

$$
u_{t}^{\epsilon}=\left(\left|D u^{\epsilon}\right|^{2}+\epsilon^{2}\right)^{1 / 2} H^{\epsilon}
$$

we deduce

$$
\begin{aligned}
\int_{0}^{\beta} \int_{R^{n}} \zeta^{2}\left|u_{t}^{\epsilon}\right| d x d t \leq & C \int_{0}^{\beta} \int_{R^{n}}\left(\zeta^{2}+|D \zeta|^{2}\right)\left(\left|D u^{\epsilon}\right|^{2}+\epsilon^{2}\right)^{1 / 2} d x d t \\
& +\int_{R^{n}} \zeta^{2}\left(|D g|^{2}+\epsilon^{2}\right)^{1 / 2} d x
\end{aligned}
$$

Recall (3.29), (3.30), (2.2) and send $\epsilon \rightarrow 0$ :

$$
\int_{0}^{\beta} \int_{B(0,1 / 2)}\left|u_{t}\right| d x d t \leq C \int_{B(0,3 / 4)}|D g| d x .
$$

(3) Assume now statment (3.28) is valid, in which case $u \leq-\theta<0$ on $B(0,1 / 2) \times$ $[\alpha, \beta]$ for some constant $\theta>0$. Fix $0<h<\theta$ and choose a smooth function $\Psi: \mathbb{R} \rightarrow \mathbb{R}$ so that

$$
\left\{\begin{array}{l}
\Psi^{\prime} \geq 0, \Psi \equiv-1 \text { on }(-\infty,-h] \\
\Phi=0 \text { on }[0, \infty),\left|\Psi^{\prime}\right| \leq \frac{C}{h}
\end{array}\right.
$$

Set

$$
\tilde{u}=\Psi(u), \tilde{g}=\Psi(g)
$$

According to [6, Theorem 2.8], $\tilde{u}$ is the (unique) weak solution of the mean curvature PDE (2.3) corresponding to the initial function $\tilde{g}$. Thus (3.31), with $\tilde{u}$ and $\tilde{g}$ replacing $u$ and $g$ gives the bound

$$
\begin{aligned}
\int_{0}^{\beta} \int_{B(0,1 / 2)}\left|\tilde{u}_{t}\right| d x d t & \leq C \int_{B(0,3 / 4)}|D \tilde{g}| d x \\
& \leq \frac{C}{h} \int_{\{-h \leq g \leq 0\} \cap B(0,3 / 4)}|D g| d x
\end{aligned}
$$

Now $\tilde{u}=\Psi(u)=-1$ on $B(0,1 / 2) \times\{t=\beta\}$ according to (3.28), and $\tilde{u}=\tilde{g}=\Psi(g)=0$ on $(B(0,1 / 2)-V) \times\{t=0\}$ according (3.25).

Since the Isoperimetric Inequality implies $|V| \leq C H^{n-1}(\partial V)^{n / n-1} \leq C \eta^{n / n-1}$, we have

$$
|\{x \in B(0,1 / 2) \mid \tilde{g}=0\}| \geq \frac{1}{2}|B(0,1 / 2)|
$$


if $\eta$ is small enough. Consequently,

$$
\begin{aligned}
\int_{0}^{\beta} \int_{B(0,1 / 2)}\left|\tilde{u}_{t}\right| d x d t & \geq \int_{\{\tilde{g}=0\} \cap B(0,1 / 2)}\left(\int_{0}^{\beta}-\tilde{u}_{t} d t\right) d x \\
& \geq \frac{1}{2}|B(0,1 / 2)| .
\end{aligned}
$$

On the other hand,

$$
\begin{aligned}
\lim _{h \rightarrow 0} \frac{1}{h} \int_{\{-h \leq g \leq 0\} \cap B(0,3 / 4)}|D g| d x & =C H^{n-1}(\{g=0\} \cap B(0,3 / 4)) \\
& \leq C H^{n-1}(\partial V) \\
& \leq C \eta
\end{aligned}
$$

This computation, combined with (3.32) and (3.33), leads to a contradiction.

\section{Estimates on extinction times and on Hausdorff measure}

This section presents two fairly straightforward applications of the "clearing out" Theorem 3.1.

4.1. Extinction time. Given a compact set $\Gamma_{0} \subset \mathbb{R}^{n}$, we as usual denote by $\left\{\Gamma_{t}\right\}_{t \geq 0}$ the subsequent generalized motion via mean curvature. Since $\Gamma_{0}$ lies within a ball of radius

$$
R \leq\left(\frac{n}{2(n+1)}\right)^{1 / 2} \operatorname{diam}\left(\Gamma_{0}\right)
$$

according to Jung's Theorem [8, Sec. 2.10.41], and this ball in turn vanishes under mean curvature flow at time $R^{2} /[2(n-1)]$ [6, Sec. 7.1], we deduce

$$
0 \leq t^{*} \leq \frac{n}{4\left(n^{2}-1\right)} \operatorname{diam}\left(\Gamma_{0}\right)^{2}
$$

Here $t^{*}$ is the extinction time for $\left\{\Gamma_{t}\right\}_{t \geq 0}$ :

$$
t^{*} \equiv \sup \left\{t \geq 0 \mid \Gamma_{t} \neq \emptyset\right\} \text {. }
$$

However, (4.1) is a gross overestimate should $\Gamma_{0}$ be, say, a long, thin ellipsoid. We are consequently interested in discovering refined upper bounds for $t^{*}$, which take into account more of the geometry of $\Gamma_{0}$. A first step in the direction is the following theorem. 
Theorem 4.1. There exists a constant $C_{1}$, depending only on $n$, such that

$$
0 \leq t^{*} \leq C_{1} H^{n-1}\left(\Gamma_{0}\right)^{2 / n-1}
$$

Estimate (4.2) is valid even though the sets $\left\{\Gamma_{t}\right\}_{t \geq 0}$ may develop an interior: see Section 4.2. It can also happen that $t^{*}=0$, even if $H^{n-1}\left(\Gamma_{0}\right)>0$ (see [6, Theorem 8.1]).

Proof. If $H^{n-1}\left(\Gamma_{0}\right)=+\infty$, there is nothing to prove. Assume instead $0<H^{n-1}\left(\Gamma_{0}\right)<$ $\infty$ and choose $r>0$ satisfying

$$
H^{n-1}\left(\Gamma_{0}\right)=\eta r^{n-1}
$$

$\eta$ the constant from Theorem 3.1. Then for each $x_{0} \in \mathbb{R}^{n}$,

$$
H^{n-1}\left(\Gamma_{0} \cap B\left(x_{0}, r\right)\right) \leq \eta r^{n-1}
$$

and so

$$
\Gamma_{t} \cap B\left(x_{0}, r / 2\right)=\emptyset \quad \text { for } \quad \alpha r^{2} \leq t \leq \beta r^{2}
$$

As this is true for each point $x_{0}$, we deduce

$$
\Gamma_{t}=\emptyset \quad \text { if } \quad t \geq \alpha r^{2}
$$

Thus

$$
t^{*} \leq \alpha r^{2}=\alpha\left(\frac{H^{n-1}\left(\Gamma_{0}\right)}{\eta}\right)^{2 / n-1}
$$

according to (4.3).

Finally, if $H^{n-1}\left(\Gamma_{0}\right)=0$, we immediately deduce $t^{*}=0$ from Theorem 3.1.

An extremely interesting open problem is to derive reasonable lower bounds on $t^{*}$ in terms of the geometry of $\Gamma_{0}$.

4.2. An estimate on $\boldsymbol{H}^{n-1}\left(\Gamma_{t}^{*}\right)$. Our intention next is to extend estimate (2.7) (asserting $H^{n-1}\left(\Gamma_{t}^{\gamma}\right) \leq H^{n-1}\left(\Gamma_{0}^{\gamma}\right)$ for a.e. $(\gamma, t)$ to an estimate on $H^{n-1}\left(\Gamma_{t}\right)$ for $t \geq 0$. In general, such a bound is impossible: as observed in [6, Sec. 8] it is possible that $\Gamma_{t}$ develops a nonempty interior 
for time $t>0$, even if $H^{n-1}\left(\Gamma_{0}\right)<\infty$. For example, let $n=2$ and $\Gamma_{0}$ be the " $\infty$ " shape as drawn:

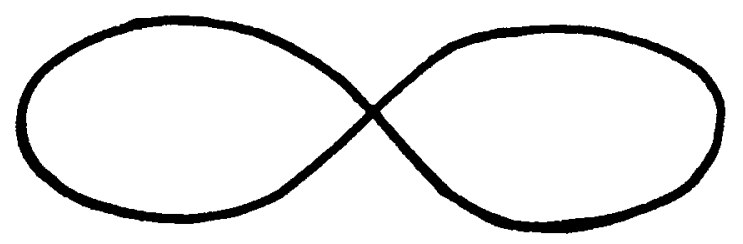

Then for each small time $t>0, \Gamma_{t}$ will look like this:

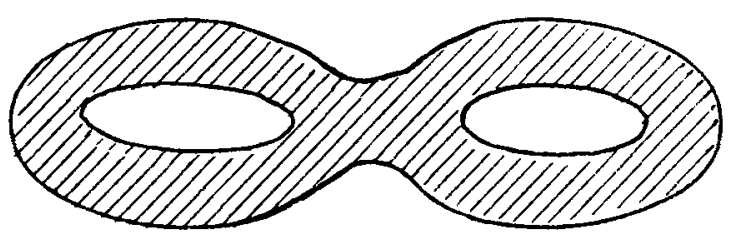

This example suggests that we turn our attention instead to the set

$$
\Gamma_{t}^{*}=\partial \Gamma_{t}
$$

the topological boundary of $\Gamma_{t}$. But note also in this example that presumably

$$
\lim _{t \backslash 0} H^{n-1}\left(\Gamma_{t}^{*}\right)=2 H^{n-1}\left(\Gamma_{0}\right)
$$

since $\Gamma_{0}$ instantly "splits" into three pieces that comprise $\Gamma_{t}^{*}$ for small time $t>0$. Consequently, the naive bound

$$
H^{n-1}\left(\Gamma_{t}^{*}\right) \leq H^{n-1}\left(\Gamma_{0}\right) \quad(t>0)
$$

suggested by (2.7), is invalid in our model.

We are, however, able to establish the following theorem.

Theorem 4.2. Assume $\Gamma_{0} \subset \mathbb{R}^{n}$ is compact and $(n-1)$-rectifiable, with $H^{n-1}\left(\Gamma_{0}\right)<\infty$. 
Then

$$
H^{n-1}\left(\Gamma_{t}^{*}\right) \leq C_{2} H^{n-1}\left(\Gamma_{0}\right) \text { for each time } t>0,
$$

the constant $C_{2}$ depending only on $n$.

Proof. (1) Let $g: \mathbb{R}^{n} \rightarrow \mathbb{R}$ be a Lipschitz function satisfying

$$
\left\{\begin{array}{l}
g(x)=\operatorname{dist}\left(x, \Gamma_{0}\right) \text { for } x \text { near } \Gamma_{0} \\
g(x) \equiv 1 \text { outside some ball, } \\
0 \leq g \leq 1 \\
\Gamma_{0}=\{g=0\}
\end{array}\right.
$$

Since $\Gamma_{0}$ is compact and $H^{n-1}$ rectifiable, Federer [8, Secs. 3.2.37 and 3.2.39] implies

$$
\lim _{r \rightarrow 0} \frac{\left|\left\{x \in \mathbb{R}^{n} \mid g(x)<r\right\}\right|}{r} \leq C H^{n-1}\left(\Gamma_{0}\right)
$$

so

$$
\int_{0}^{r} H^{n-1}\left(\Gamma_{0}^{\gamma}\right) d \gamma \leq C r H^{n-1}\left(\Gamma_{0}\right)
$$

for all sufficiently small $r>0$.

This inequality and (2.7) imply there exists a set $G \subset(0,1)$ so that

$$
\text { If } \gamma \in G, H^{n-1}\left(\Gamma_{t}^{\gamma}\right) \leq H^{n-1}\left(\Gamma_{0}^{\gamma}\right) \leq C H^{n-1}\left(\Gamma_{0}\right) \text { for a.e. } t \geq 0
$$

and

$$
|G \cap(0, r)|>\frac{r}{2} \text { for each } r>0
$$

Let $u$ be the unique weak solution of the mean curvature PDE (2.7) corresponding to the initial function $g$. Then

$$
\Gamma_{t}=\left\{x \in \mathbb{R}^{n} \mid u(x, t)=0\right\} \text { for each } t \geq 0
$$

(2) Fix a time $t>0$ and a number $s>0$. Since $\Gamma_{t}^{*}$ is compact, we can find a finite collection $\left\{U\left(x_{i}, s\right)\right\}_{i=1}^{M}$ of open balls such that

$$
x_{i} \in \Gamma_{t}^{*} \quad(i=1, \ldots, M)
$$


and

$$
\Gamma_{t}^{*} \subset \bigcup_{i=1}^{M} U\left(x_{i}, x\right)
$$

Using Vitali's Covering Theorem, we find an integer $N \leq M$ such that, upon reindexing if necessary, we may assume the closed balls $\left\{B\left(x_{i}, s\right)\right\}_{i=1}^{N}$ are disjoint, with

$$
\Gamma_{t}^{*} \subset \bigcup_{i=1}^{N} B\left(x_{i}, 5 s\right)
$$

(3) In view of (4.9), (4.8) and the continuity of $u \geq 0$, we can select an index $\gamma \in G$ satisfying

$$
\Gamma_{t}^{\gamma} \cap B\left(x_{i}, \frac{s}{2}\right) \neq \emptyset \quad(i=1, \ldots, N)
$$

Let $\alpha, \beta>0$ be the constants from Theorem 3.1. Given $\gamma$ as above, select $\alpha \leq \sigma \leq \beta$ so that

$$
H^{n-1}\left(\Gamma_{\tau}^{\gamma}\right) \leq C H^{n-1}\left(\Gamma_{0}\right)
$$

for $\tau=t-\sigma s^{2}$ : this is possible owing to (4.7).

Now we claim

$$
\eta s^{n-1} \leq H^{n-1}\left(\Gamma_{\tau}^{\gamma} \cap B\left(x_{i}, s\right)\right) \quad(i=1, \ldots, N) .
$$

For if not, then

$$
\Gamma_{t}^{\gamma} \cap B\left(x_{i}, \frac{s}{2}\right)=\emptyset
$$

according to Theorem 3.1. This assertion contradicts (4.11).

(4) Consequently, we may compute

$$
\begin{aligned}
H_{5 s}^{n-1}\left(\Gamma_{t}^{*}\right) & \leq C \sum_{i=1}^{N}(5 s)^{n-1} \text { by }(4.10) \\
& \leq C \sum_{i=1}^{N} H^{n-1}\left(\Gamma_{\tau}^{\gamma} \cap B\left(x_{i}, s\right)\right) \text { by }(4.13) \\
& \leq C H^{n-1}\left(\Gamma_{\tau}^{\gamma}\right) \\
& \leq C H^{n-1}\left(\Gamma_{0}\right) \text { by }(4.12)
\end{aligned}
$$


The constant $C$ depends only on $n$ and not on $s$. Thus sending $s \rightarrow 0^{+}$above, we deduce assertion (4.5).

\section{Local smoothness for graphs}

In this section we will establish the smoothness of our generalized mean curvature flow wherever the motion can be described locally in space and time as the graph of a continuous function. Besides being a basic regularity assertion that we hope will be useful for a general theory, this gives a complete proof of the smoothness of the motion in many interesting special cases.

5.1. The motion of graphs and weak solutions. Let us suppose that $u$ is a weak solution of our mean curvature PDE

$$
u_{t}=\left(\delta_{i j}-\frac{u_{x_{i}} u_{x_{j}}}{|D u|^{2}}\right) u_{x_{i} x_{j}} \quad \text { in } \mathbb{R}^{n} \times(0, \infty),
$$

and that in an open region $U \times\left(t_{1}, t_{2}\right) \subset \mathbb{R}^{n} \times \mathbb{R}$, the set $\Gamma=\{u=0\}$ is the graph of a continuous function $v$. Thus, say,

$$
\{(x, t) \mid u(x, t)=0\} \cap U=\left\{(x, t) \mid x^{\prime} \in U^{\prime}, x_{n}=v\left(x^{\prime}, t\right)\right\}
$$

where $x^{\prime}=\left(x_{1}, \ldots, x_{n-1}\right), U^{\prime}=U \cap\left\{x_{n}=0\right\}$, and $v$ is continuous on $U^{\prime} \times\left(t_{1}, t_{2}\right)$.

Theorem 5.1. The function $v$ is a weak solution of the PDE

$$
v_{t}=\left(\delta_{i j}-\frac{v_{x_{i}} v_{x_{j}}}{1+|D v|^{2}}\right) v_{x_{i} x_{j}} \text { in } U^{\prime} \times\left(t_{1}, t_{2}\right)
$$

(Note carefully that the implicit summation here is from 1 to $n-1$.)

Proof. (1) Let $\psi \in C^{\infty}\left(\mathbb{R}^{n-1} \times \mathbb{R}\right)$ and suppose that $v-\psi$ has a local maximum at a point $\left(x_{0}, t_{0}\right) \in U^{\prime} \times\left(t_{1}, t_{2}\right)$. We must show

$$
\psi_{t} \leq\left(\delta_{i j}-\frac{\psi_{x_{i}} \psi_{x_{j}}}{1+|D \psi|^{2}}\right) \psi_{x_{i} x_{j}} \quad \text { at } \quad\left(x_{0}, t_{0}\right)
$$

(2) We may assume that $\psi\left(x_{0}, t_{0}\right)=v\left(x_{0}, t_{0}\right)=0$; so that

$$
v\left(x^{\prime}, t\right) \leq \psi\left(x^{\prime}, t\right) \quad \text { in } \quad U^{\prime} \times\left(t_{1}, t_{2}\right)
$$


(3) Replacing $u$ by $-|u|$ if necessary (still a weak solution of (1)), we may also assume

$$
u \leq 0 \text { in } U \times\left(t_{1}, t_{2}\right)
$$

Write

$$
\phi(x, t) \equiv \psi\left(x^{\prime}, t\right)-x_{n}
$$

(4) Let

$$
\begin{aligned}
E_{k} & \equiv\left\{(x, t) \in U \times\left(t_{1}, t_{2}\right) \mid \frac{1}{2^{k}} \leq x_{n}-\psi\left(x^{\prime}, t\right) \leq \frac{1}{2^{k-1}}\right\} \quad(k=1,2, \ldots) \\
E_{0} & =\left\{(x, t) \in U \times\left(t_{1}, t_{2}\right) \mid 1 \leq x_{n}-\psi\left(x^{\prime}, t\right)\right\}
\end{aligned}
$$

Define

$$
\alpha_{k} \equiv \sup \left\{u(x, t) \mid(x, t) \in \bigcup_{0 \leq j \leq k} E_{j}\right\}
$$

Then by (5.2) and (5.6),

$$
\alpha_{k}<0, \lim _{k \rightarrow \infty} \alpha_{k}=0, \alpha_{0} \leq \alpha_{1} \leq \alpha_{2} \leq \cdots \leq \alpha_{k} \leq \cdots<0
$$

We reindex as necessary to obtain

$$
\alpha_{0}<\alpha_{1}<\alpha_{2}<\cdots<\alpha_{k}<\cdots<0, \lim _{k \rightarrow \infty} \alpha_{k}=0
$$

(5) Define $\Phi: \mathbb{R} \rightarrow \mathbb{R}$ by setting

$$
\left\{\begin{array}{l}
\Phi\left(\alpha_{k}\right)=-\frac{1}{2^{k-1}} \quad(k=0,1, \ldots) \\
\Phi \text { linear on }\left[\alpha_{k}, \alpha_{k+1}\right] \\
\Phi(0)=0, \Phi(r)=r, r>0 \\
\Phi(r)=-2, r \leq \alpha_{0}
\end{array}\right.
$$

Then if $(x, t) \in E_{k}, u(x, t) \leq \alpha_{k}$ and so

$$
\Phi(u(x, t)) \leq \Phi\left(\alpha_{k}\right)=-\frac{1}{2^{k-1}} \leq \psi\left(x^{\prime}, t\right)-x_{n}=\phi(x, t) .
$$

Thus

$$
\Phi(u(x, t)) \leq \phi(x, t) \quad \text { on } \quad \bigcup_{k=0}^{\infty} E_{k}=\left\{(x, t) \in U \times\left(t_{1}, t_{2}\right) \mid x_{n}>\psi\left(x^{\prime}, t\right)\right\} .
$$


On $\left\{(x, t) \in U \times\left(t_{1}, t_{2}\right) \mid x_{n} \leq \psi\left(x^{\prime}, t\right)\right\}$, we have

$$
\Phi(u(x, t)) \leq 0 \leq \phi(x, t) .
$$

Consequently, $w(x, t) \equiv \Phi(u(x, t)) \leq \phi(x, t)$ in $U \times\left(t_{1}, t_{2}\right), w\left(x_{0}, t_{0}\right)=\phi\left(x_{0}, t_{0}\right)$. Therefore $w-\phi$ has a local maximum in $U \times\left(t_{1}, t_{2}\right)$ at $\left(x_{0}, t_{0}\right)$. Since $w$ is a weak solution of (5.1) and $D \phi\left(x_{0}, t_{0}\right) \neq 0$, we have

$$
\phi_{t} \leq\left(\delta_{i j}-\frac{\phi_{x_{i}} \phi_{x_{j}}}{|D \phi|^{2}}\right) \phi_{x_{i} x_{j}} \quad \text { at }\left(x_{0}, t_{0}\right)
$$

But

$$
\begin{array}{rl}
\phi_{t}=\psi_{t}, & \phi_{x_{n}}=-1 \\
\phi_{x_{i}}=\psi_{x_{i}} & 1 \leq i \leq n-1 \\
\phi_{x_{i} x_{j}}=\psi_{x_{i} x_{j}} & 1 \leq i, j \leq n-1 \\
0 & \text { otherwise. }
\end{array}
$$

Hence (5.8) implies (5.4).

(6) Similarly, if $v-\psi$ has a local minimum at $\left(x_{0}, t_{0}\right) \in U^{\prime} \times\left(t_{1}, t_{2}\right)$,

$$
\psi_{t} \geq\left(\delta_{i j}-\frac{\psi_{x_{i}} \psi_{x_{j}}}{1+|D \psi|^{2}}\right) \psi_{x_{i} x_{j}} \quad \text { at }\left(x_{0}, t_{0}\right)
$$

Therefore $v$ is a weak solution of (5.3).

5.2. Interior gradient bounds. Our strategy now is to construct a smooth solution of the evolution equation (5.3) for arbitrary continuous initial and boundary data. This will be accomplished in the next section via an approximation argument. In order to obtain a smooth solution, it is necessary to have a suitable a priori interior gradient estimate for smooth solutions of

$$
v_{t}=\left(\delta_{i j}-\frac{v_{x_{i}} v_{x_{j}}}{1+|D v|^{2}}\right) v_{x_{i} x_{j}}
$$

It is convenient first to consider negative solutions of (5.10). Set $\Omega_{T} \equiv B(0, R) \times(0,2 T)$. Adapting the method of Korevaar [11] we have

Theorem 5.2. Suppose $v \in C^{3}\left(\Omega_{T}\right) \cap C^{0}\left(\bar{\Omega}_{T}\right)$ is a negative solution of (5.10) with $v(0, T)=-v_{0}$. Then

$$
|D v(0, T)| \leq\left(3+16 \frac{v_{0}}{R}\right) e^{2 K}
$$

where $K \equiv 2+20\left(v_{0}^{2} / T\right)+80 n\left(v_{0}^{2} / R^{2}\right)$. 
Corollary 5.3. Let $v \in C^{3}\left(\Omega_{T}\right) \cap C^{0}\left(\bar{\Omega}_{T}\right)$ be an arbitrary solution of (5.10). Then

$$
|D v(0, T)| \leq\left(3+48 \frac{M}{R}\right) e^{2 \bar{K}}
$$

where $\bar{K} \equiv 2+180\left(M^{2} / T\right)+720 n\left(M^{2} / R^{2}\right), M \equiv \sup _{\Omega_{T}}|v|$.

Proof of Theorem 5.2. (1) Assume first $R=1$ and set

$$
w \equiv \sqrt{1+|D v|^{2}} \quad v^{i}=\frac{v_{x_{i}}}{w}, g^{i j} \equiv \delta_{i j}-\nu^{i} \nu^{j} \quad(i, j=1, \ldots, n)
$$

Define $h(x, t) \equiv \eta(x, t, v(x, t)) w$ in $\Omega_{T}$, where $\eta(x, t, z)$ is nonnegative, vanishes on the set $\left\{t\left(1-|x|^{2}\right)=0\right\}$, and is smooth where it is positive. Then $h$ is nonnegative and vanishes on the parabolic boundary of $\Omega_{T}$.

(2) We compute $L h \equiv g^{i j} h_{x_{i} x_{j}}-h_{t}$, using parentheses around $\eta$ to denote total derivatives. Thus

$$
\begin{aligned}
h_{t} & =\eta w_{t}+w(\eta)_{t} \\
h_{x_{i}} & =\eta w_{x_{i}}+w(\eta)_{x_{i}} \\
h_{x_{i} x_{j}} & =\eta w_{x_{i} x_{j}}+w_{x_{i}}(\eta)_{x_{j}}+w_{x_{j}}(\eta)_{x_{i}}+w(\eta)_{x_{i} x_{j}}
\end{aligned}
$$

Hence

$$
L h=\eta L w+2 g^{i j} \frac{w_{x_{i}}}{w} h_{x_{j}}-\frac{2 \eta}{w} g^{i j} w_{x_{i}} w_{x_{j}}+w L \eta
$$

so that

$$
L h-2 g^{i j} \frac{w_{x_{i}}}{w} h_{x_{j}}=\eta\left(L w-\frac{2}{w} g^{i j} w_{x_{i}} w_{x_{j}}\right)+w L \eta
$$

(3) We next show

$$
L w-\frac{2}{w} g^{i j} w_{x_{i}} w_{x_{j}} \geq 0
$$

Indeed,

$$
w_{x_{i}}=\nu^{k} v_{x_{k} x_{i}}
$$

and

$$
w_{x_{i} x_{j}}=\nu^{k} v_{x_{k} x_{i} x_{j}}+\frac{1}{w}\left(v_{x_{k} x_{i}} v_{x_{k} x_{j}}-\nu^{k} v_{x_{k} x_{i}} v_{v_{x_{\ell} x_{j}}}^{\ell}\right)
$$


From (5.13) and (5.16), we deduce

$$
g^{i j} w_{x_{i} x_{j}} \geq \nu^{k} g^{i j} v_{x_{k} x_{i} x_{j}}
$$

But

$$
\begin{aligned}
\nu^{k} g^{i j} v_{x_{k} x_{i} x_{j}} & =\nu^{k}\left(\left(g^{i j} v_{x_{i} x_{j}}\right)_{x_{k}}-g_{x_{k}}^{i j} v_{x_{i} x_{j}}\right) \\
& =\nu^{k} v_{t x_{k}}+\nu^{k} \frac{v_{x_{i} x_{j}}}{w}\left(\left(v_{x_{i} x_{k}} \nu^{j}+v_{x_{j} x_{k}} \nu^{i}\right)-2 \frac{v_{x_{i}} v_{x_{j}}}{w^{2}} w_{x_{k}}\right) \\
& =w_{t}+\frac{2}{w} g^{i j} w_{x_{i}} w_{x_{j}} .
\end{aligned}
$$

Combining (5.17) and (5.18) gives (5.15), as claimed. Now (5.14) and (5.15) imply

$$
\tilde{L} h \equiv L h-2 g^{i j} \frac{w_{x_{i}}}{w} h_{x_{j}} \geq w L \eta
$$

(4) We choose $\eta=f \circ \phi(x, t, v(x, t))$, where

$$
\phi(x, t, z)=\left(\frac{z}{2 v_{0}}+\frac{t}{T}\left(1-|x|^{2}\right)\right)^{+}, f(\phi)=e^{K \phi}-1
$$

On the set where $\phi>0$,

$$
\left\{\begin{array}{l}
\phi_{z}=\frac{1}{2 v_{0}}, \phi_{t}=\frac{1-|x|^{2}}{T}, \phi_{z z}=\phi_{x_{i} z}=0 \\
\phi_{x_{i} x_{j}}=-\frac{2 t}{T} \delta_{i j} \\
0 \leq \phi \leq 2, \Sigma \phi_{x_{i}}^{2} \leq \frac{4 t^{2}}{T^{2}} \leq 16
\end{array}\right.
$$

Thus on the set where $h(x, t)>0$,

$$
\begin{aligned}
(\eta)_{t} & =f^{\prime}\left(\phi_{t}+\phi_{z} v_{t}\right),(\eta)_{x_{i}}=f^{\prime}\left(\phi_{x_{i}}+\phi_{z} v_{x_{i}}\right) \\
(\eta)_{x_{i} x_{j}} & =f^{\prime \prime}\left(\phi_{x_{i}}+\phi_{z} v_{x_{i}}\right)\left(\phi_{x_{j}}+\phi_{z} v_{x_{j}}\right)+f^{\prime}\left(\phi_{x_{i} x_{j}}+\phi_{z} v_{x_{i} x_{j}}\right)
\end{aligned}
$$

Consequently,

$$
\begin{aligned}
L \eta= & f^{\prime \prime} g^{i j}\left(\phi_{x_{i}}+\phi_{z} v_{x_{i}}\right)\left(\phi_{x_{j}}+\phi_{z} v_{x_{j}}\right) \\
& +f^{\prime}\left(g^{i j} \phi_{x_{i} x_{j}}-\phi_{t}\right)+f^{\prime} \phi_{z} L v .
\end{aligned}
$$

As $L v=0$ and the least eigenvalue of the matrix $\left(g^{i j}\right)$ is $1 / w^{2},(5.21)$ implies

$$
L \eta \geq \frac{f^{\prime \prime}}{w^{2}} \Sigma\left(\phi_{x_{i}}+\phi_{z} v_{x_{i}}\right)^{2}+f^{\prime}\left(g^{i j} \phi_{x_{i} x_{j}}-\phi_{t}\right)
$$


Using (5.20), we find

$$
\begin{aligned}
g^{i j} \phi_{x_{i} x_{j}}-\phi_{t} & =-\frac{1}{T}\left(2 t\left(n-\frac{|D v|^{2}}{w^{2}}\right)+\left(1-|x|^{2}\right)\right) \geq-\left(4 n+\frac{1}{T}\right), \\
\Sigma\left(\phi_{x_{i}}+\phi_{z} v_{x_{i}}\right)^{2} & \geq \frac{1}{8 v_{0}^{2}}|D v|^{2}-16 \geq \frac{1}{16 v_{0}^{2}}|D v|^{2} \geq \frac{w^{2}}{20 v_{0}^{2}}
\end{aligned}
$$

provided $|D v| \geq \max \left(16 v_{0}, 2\right)$. Thus, by our choice of $f,(5.22)$ gives

$$
L \eta \geq K e^{K \phi}\left\{\frac{1}{20 v_{0}^{2}} K-\left(4 n+\frac{1}{T}\right)\right\}
$$

on the set $\left\{h>0,|D v|>\max \left(16 v_{0}, 2\right)\right\}$. Choosing $K=2+20\left(v_{0}^{2} / T\right)+80 n v_{0}^{2}$, we deduce from (5.19) and (5.24) that

$$
\tilde{L} h \geq 0 \text { on }\left\{h>0,|D v|>\max \left(16 v_{0}, 2\right)\right\}
$$

Therefore (5.25) and the maximum principle give

$$
h(0, T)=\left(e^{K / 2}-1\right) w(0, T) \leq \max h \leq\left(e^{2 K}-1\right) \max \left(\sqrt{5}, \sqrt{1+\left(16 v_{0}\right)^{2}}\right) .
$$

This estimate can be simplified to read

$$
w(0, T) \leq\left(3+16 v_{0}\right) e^{2 K}, \quad K=2+20 \frac{v_{0}^{2}}{T}+80 n v_{0}^{2} .
$$

This proves (5.11) for $R=1$.

(5) Finally, the case of arbitrary $R$ is recovered from (5.11) via the scaling

$$
v \rightarrow \frac{1}{R} v\left(R x, R^{2} t\right)
$$

defined on $B_{1}(0) \times\left(0,2 T / R^{2}\right)$.

5.3. Regularity of the height function. We are now ready to prove our main regularity assertion. 
Theorem 5.4. Let $\left\{\Gamma_{t}\right\}_{t \geq 0}$ denote the generalized evolution by mean curvature corresponding to some compact set $\Gamma_{0} \subset \mathbb{R}^{n}$. Suppose that in a neighborhood $U \times\left(t_{0}-\epsilon, t_{0}+\epsilon\right)$ of $\left(x_{0}, t_{0}\right)$ the set $\Gamma$ is a continuous graph. Then $\Gamma \cap U$ is a $C^{\infty}$ graph.

Proof. As in Section 5.1, we may suppose (see 5.2)

$$
\begin{aligned}
\Gamma_{t} \cap U & =\{x \mid u(x, t)=0\} \cap U \\
& =\left\{\left(x^{\prime}, x_{n}\right) \mid x^{\prime} \in U^{\prime}, x_{n}=v\left(x^{\prime}, t\right)\right\} \quad\left(t_{0}-\epsilon<t<t_{0}+\epsilon\right)
\end{aligned}
$$

where $U^{\prime}=U \cap\left\{x_{n}=0\right\}$. Without loss of generality, we take $U^{\prime}=U(0,1) \subset R^{n-1}$. According to Theorem 5.1, $v$ is then a weak solution of the PDE

$$
v_{t}=\left(\delta_{i j}-\frac{v_{x_{i}} v_{x_{j}}}{1+|D v|^{2}}\right) v_{x_{i} x_{j}} \quad \text { in } \quad U(0,1) \times\left(t_{0}-\epsilon, t_{0}+\epsilon\right)
$$

subject to the boundary conditions

$$
\left\{\begin{array}{l}
v\left(x^{\prime}, t_{0}-\epsilon\right)=v_{0}\left(x^{\prime}\right) \text { on } U(0,1) \\
v=\varphi\left(x^{\prime}, t\right) \quad \text { on } \quad \partial U(0,1) \times\left(t_{0}-\epsilon, t_{0}+\epsilon\right)
\end{array}\right.
$$

with $v_{0}$ and $\varphi$ continuous. Using the technique of [6, Sec. 3.2], we know that $v$ is the unique weak solution of (5.27), (5.28). Thus the proof of the theorem is reduced to showing that the initial boundary value problem (5.27), (5.28) possesses a classical solution

$$
v \in C^{\infty}(\Omega) \cap C^{0}(\bar{\Omega}), \Omega \equiv U(0,1) \times\left(t_{0}-\epsilon, t_{0}+\epsilon\right) .
$$

For smooth $v_{0}, \varphi$ this is proven in Lieberman [13, p. 385]. Moreover, this technique of Lieberman [13, Sec. 2] and Gilbarg and Trudinger [9, Sec. 14.5] give moduli of continuity estimates for $v$ when $v_{0}, \varphi$ are merely continuous.

Choose now smooth functions $v_{0}^{k}, \varphi^{k}$ converging uniformly to $v_{0}, \varphi$ on $U(0,1)$, respectively $\partial U(0,1) \times\left[t_{0}-\epsilon, t_{0}+\epsilon\right]$, with corresponding solutions $v^{k}$ of (5.27). Then by Corollary 7.3 (with $n$ replaced by $n-1)\left\{\left|D v^{k}\right|\right\}_{k=1}^{\infty}$ is uniformly bounded on compact subsets $Q$ of $U(0,1) \times$ $\left(t_{0}-\epsilon, t_{0}+\epsilon\right)$. Thus each $v^{k}$ satisfies a uniformly parabolic equation, wherein classic interior Holder gradient and Schauder estimates (cf. [12]) imply that

$$
\sup _{k}\left\|v^{k}\right\|_{C^{2}+\alpha, \frac{2+\alpha}{2}(\bar{Q})}<\infty
$$

Thus, passing to a subsequence if necessary, $\left\{v^{k}\right\}_{k=1}^{\infty}$ converges to a classic $C^{\infty}$ solution $v$ of (5.27). Moreover, $v$ satisfies the initial and boundary conditions (5.28) by virtue of the modulus 
of continuity estimates mentioned earlier. Therefore we have shown

$$
\Gamma \cap U=\left\{(x, t) \mid x^{\prime} \in U(0,1), x_{n}=v\left(x^{\prime}, t\right)\right\}
$$

is the graph of a $C^{\infty}$ function.

5.4. More on convexity. As an immediate application of Theorem 5.4 we can improve our previous result $\left[6\right.$, Theorem 7.6] concerning the evolution of convex initial hypersurfaces $\Gamma_{0}$. These assertions recover by our methods some of Huisken's results in [10].

Theorem 5.5. Assume $\Gamma_{0}$ is the boundary of a convex, bounded, open set $U$. Then there exists a time $t^{*}>0$ such that $\Gamma_{t}$ is the boundary of a smooth convex, nonempty open set for $0 \leq t<t^{*}$, and is empty for $t>t_{*}$.

Proof. Choose admissible initial data $g$ such that $\Gamma_{0} \equiv\{g=0\}, g<0$ outside $U$, $g>0$ inside $U$ and the level sets $\{x \in U \mid g(x)= \pm \gamma\}$ for small $\gamma>0$ are smooth and strictly convex. Let $u$ be the corresponding solution of our mean curvature evolution PDE (1.1). Applying [6, Theorem 7.6] and Theorem 5.4, we conclude that the sets $\{x \mid u(x, t)>\gamma\}$, $\{x \mid u(x, t)>-\gamma\}$ for small $\gamma>0$ are smooth and convex. Since intersections and increasing unions of convex sets are convex, it follows that for each $t>0$, the sets $L(t) \equiv\{x \mid u(x, t)>$ $0\}$ and $U(t) \equiv\{x \mid u(x, t) \geq 0\}$ are convex. In particular, $\partial \bar{L}(t)$ and $\partial U(t)$ are Lipschitz and coincide, unless $\Gamma_{t}$ has nonempty interior. However, this last possibility is excluded by the uniqueness result of Soner $[18$, Secs. 7,9$]$ since $\Gamma_{0}$ is Lipschitz and strictly star-shaped. It follows that

$$
\Gamma_{t}=\partial \bar{L}(t)=\partial U(t)
$$

Now fix a point $\left(x_{0}, t_{0}\right)$ with $x_{0} \in \Gamma_{t_{0}}, t_{0}<t^{*}=$ the extinction time for $\Gamma_{0}$. By the previous reasoning, we can find a neighborhood $\mathcal{O}$ of $\left(x_{0}, t_{0}\right)$ so that we can represent

$$
\Gamma \cap \mathcal{O}=\left\{(x, t) \mid x^{\prime} \in U(0, r), x_{n}=v\left(x^{\prime}, t\right)\right\}
$$

where $v$ is Lipschitz in $x^{\prime}$ uniformly in $t$ and the $x_{n}$ direction is chosen appropriately with respect to a fixed ball contained inside the $\Gamma_{t}$. In order to apply Theorem 5.4, it remains to demonstrate the continuity of $v$ in $t$. Consider a sequence $\left(x_{k}^{\prime}, t_{k}\right)$ in $U(0, r / 2) \times\left(t_{0}-\epsilon / 2, t_{0}+\epsilon / 2\right)$ converging to point $\left(\bar{x}^{\prime}, \bar{t}\right)$ in $U(0, r) \times\left(t_{0}-\epsilon, t_{0}+\epsilon\right)$. Since $v$ is bounded, we can choose a subsequence $\left(x_{k_{i}}^{\prime}, t_{k_{i}}\right)$ so that $v\left(x_{k_{i}}^{\prime}, t_{k_{i}}\right) \rightarrow L$. On the other hand, $\left.u\left(\left(x_{k_{i}}^{\prime}, v\left(x_{k_{i}}^{\prime}, t_{k_{i}}\right)\right), t_{k_{i}}\right), t_{k_{i}}\right)=0$ and 
$u$ is continuous. Therefore, $u\left(\left(x^{\prime}, L\right), \bar{t}\right)=0$ and hence $L=v\left(\bar{x}^{\prime}, \bar{t}\right)$ by our representation of $\Gamma \cap \mathcal{O}$.

Hence $v$ is continuous and Theorem 5.4 implies that $\Gamma_{t}$ is smooth and convex for all $t<t^{*}$.

Example. Consider $\Gamma_{0}$ consisting of a circle $C$ in the plane union a vertical diameter as drawn:

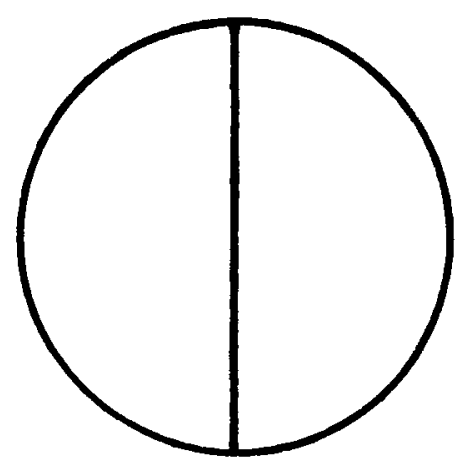

Then for each small time $t>0, \Gamma_{t}$ will look like this:

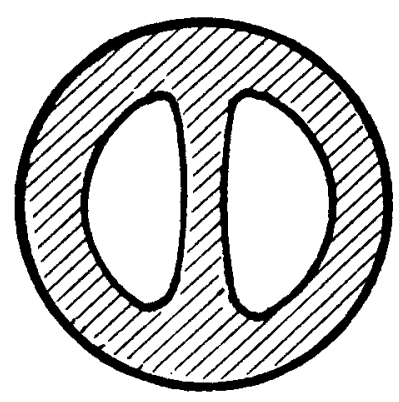

To see this heuristically, consider the left closed semicircle $\Sigma$ as a subset of $\Gamma_{0}$. By [6, Theorem 7.2], $\Sigma_{t} \subseteq \Gamma_{t}$ for each $t>0$. According to Theorem 5.5, $\Sigma_{t}$ is smooth and convex. Similarly, $\Gamma_{0}$ contains the motion $C_{t}$ of the circle $C$. On the other hand, $\Sigma_{t}$ must separate immediately from $C_{t}$ and be contained inside it [6, Theorem 8.2]. By symmetry, the same situation 
pertains to the right closed semicircle. Thus $\Gamma_{t}$ must have nonempty interior and look as drawn above.

Besides giving a nice example of the development of an interior, this example shows the hypothesis of Theorem 5.4 that $\Gamma_{t}$ be representable locally as a graph for an interval of time is necessary for regularity.

\section{References}

[1] Allard, W. On the first variation of a varifold. Ann. Math. 95, 417-491 (1972).

[2] Brakke, K. A. The Motion of a Surface by its Mean Curvature. Princeton, NJ: Princeton University Press 1978.

[3] Burago, Y. D., and Zalgaller, V. A. Geometric Inequalities. New York: Springer-Verlag 1988.

[4] Chen, Y.-G., Giga, Y., and Goto, S. Uniqueness and existence of viscosity solutions of generalized mean curvature flow equations. Preprint.

[5] Ecker, E., and Huisken, G. Mean curvature evolution of entire graphs. Ann. Math. 130, 453-471 (1989).

[6] Evans, L. C., and Spruck, J. Motion of level sets by mean curvature I. J. Diff. Geom. 33, 635-681 (1991).

[7] Evans, L. C., and Spruck, J. Motion of level sets by mean curvature II. Trans. AMS. To appear.

[8] Federer, H. Geometric Measure Theory. New York: Springer-Verlag 1969.

[9] Gilbarg, D., and Trudinger, N. S. Elliptic Partial Differential Equations of Second Order. New York: Springer-Verlag 1983.

[10] Huisken, G. Flow by mean curvature of convex surfaces into spheres. J. Diff. Geom. 20, 237-266 (1984).

[11] Korevaar, N. J. An easy proof of the interior gradient bound for solutions to the prescribed mean curvature equation. Proc. Symposia Pure Math. 45 (1986).

[12] Ladyzhenskaja, O. A., Solonnikov, and Ural'tseva, N. N. Linear and Quasi-Linear Equations of Parabolic Type. Providence, RI: American Mathematical Society 1968.

[13] Lieberman, G. M. The first initial-boundary value problem for quasilinear second order parabolic equations. Ann. Scuola Norm. Sup. Pisa 13, 347-387 (1986).

[14] Michael, J. H., and Simon, L. M. Sovolev and mean value inequalities on generalized submanifolds of $R^{n}$. Comm. Pure Appl. Math. 26, 361-379 (1973).

[15] Osher, S., and Sethian, J. A. Fronts propagating with curvature dependent speed: Algorithms based on HamiltonJacobi formulations. J. Comput. Phys. 79, 12-49 (1988).

[16] Sethian, J. A. Numerical algorithms for propagating interfaces: Hamilton-Jacobi equations and conservation laws. J. Diff. Geom. 31, 131-161 (1990).

[17] Mete Soner, $\mathrm{H}$. Motion of a set by the curvature of its boundary. Preprint.

[18] Stein, E. M. Singular Intervals and Differentiability Properties of Functions. Princeton, NJ: Princeton University Press 1970. 